Article

\title{
Control Methods for Single-phase Voltage Supply with VSCs to Feed Nonlinear Loads in Rural Areas*
}

\author{
Walter Gil-González ${ }^{1 凶 \mathbb{D}}$, Sara Yulieth Bocanegra ${ }^{2}$, Federico. M. Serra ${ }^{3 凶 \mathbb{D}}$, Maximiliano

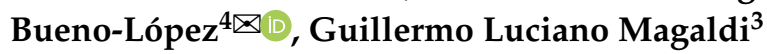 \\ 1 Grupo GIIEN, Facultad de Ingeniería, Institución Universitaria Pascual Bravo, Campus Robledo, 050036, \\ Medellín, Colombia. \\ 2 Estudiante de Maestría en Ingeniería Eléctrica, Universidad Tecnológica de Pereira, 660003, Pereira, Colombia. \\ 3 Laboratorio de Control Automático, Universidad Nacional de San Luis - Postcode: 5730 - Villa Mercerdes, \\ Argentina. \\ 4 Docente en el Departamento de Electrónica, Instrumentación y Control. Universidad del Cauca. Popayán, \\ Colombia.. \\ $\bigotimes_{\text {walter.gil@pascualbravo.edu.co }}$
}

Received: 13 February 2020; Accepted: 27 March 2020; Published: 17 December 2020

\begin{abstract}
This paper addresses the problem of sinusoidal voltage generation in linear loads using a voltage source inverter (VSI). The port-Hamiltonian structure in open-loop is used to design a passivity-based controller with proportional-integral gains (PI-PBC) in order to develop the control strategy. The main advantage of using passivity-based controllers corresponds to the possibility of guaranteeing asymptotic stability by transforming the trajectory tracking problem into a regulation control one. In addition to the PI-PBC, a linear load estimator is employed based on an integral formulation to determine the value of the equivalent conductance in the load, which reduces the number of current sensors. Numerical validations demonstrate that the sinusoidal voltage provided by the VSI to the load has a tracking error lower than $1 \%$, with harmonic distortions lower than $2.6 \%$, both for voltage and currents in the load. All the simulations were conducted in MATLAB/Simulink using the SimPowerSystems library version 2017a.
\end{abstract}

Keywords: Linear loads, passivity-based control, stability analysis, voltage generation, voltage source converters.

Resumen: Este artículo aborda el problema de la generación de voltaje sinusoidal en cargas lineales utilizando un inversor de fuente de voltaje (VSI). La estructura port-Hamiltoniana en lazo abierto se utiliza para diseñar un controlador basado en pasividad con ganancias proporcionales-integrales (PI-PBC) con el fin de desarrollar la estrategia de control. La principal ventaja de utilizar controladores basados en pasividad corresponde a la posibilidad de garantizar la estabilidad asintótica transformando el problema de seguimiento de trayectoria en uno de control de regulación. Además del PI-PBC, se emplea un estimador de carga lineal basado en una formulación integral para determinar el valor de la conductancia equivalente en la carga, lo que reduce el número de sensores de corriente. Las validaciones

\footnotetext{
${ }^{*}$ How to cite this article: Gil Gonzalez, W., Bocanegra, S., Serra, F., Bueno-López, M., Magaldi, G. (2020). Control Methods for Single-phase Voltage Supply with VSCs to Feed Nonlinear Loads in Rural Areas. Transactions on Energy Systems and Engineering Applications, 1(1), 33-47. DOI: 10.32397/tesea.vol1.n1.3 ISSN: 2745-0120
} 
numéricas demuestran que el voltaje sinusoidal proporcionado por el VSI a la carga tiene un error de seguimiento menor a $1 \%$, con distorsiones armónicas menores a $2.6 \%$, tanto para voltaje como para corrientes en la carga. Todas las simulaciones se realizaron en MATLAB/Simulink utilizando la biblioteca SimPowerSystems versión 2017 a.

Palabras claves: Cargas lineales, control basado en pasividad, análisis de estabilidad, generación de voltaje, convertidores de fuente de voltaje.

\section{Introducción}

\subsection{Contexto general}

Las redes de distribución de energía eléctrica son consideradas como un elemento esencial en la vida de los seres humanos ya que, a partir de su aparición, estas se han convertido en en un factor fundamental para el desarrollo económico y social en centros urbanos y zonas rurales aledañas a estos (Abu-Rub et al. 2014; Ellabban et al. 2014; Montoya et al. 2019b; Parhizi et al. 2015). Sin embargo, las comunidades que se encuentran alejadas de las redes eléctricas convencionales, se ven relegadas a un segundo plano sin la posibilidad de acceso a tecnología, redes de comunicación o Internet; incluso en algunos casos sufren la ausencia de servicios esenciales como salud y educación, los cuales son derechos fundamentales y que hacen parte de una vida digna (Bueno-Lopez and Garzon Lemos 2017). Por lo anterior, una prioridad de los gobiernos nacionales es garantizar la cobertura del servicio de electricidad a toda su población, ya que está viene ligada al desarrollo socio-económico de las comunidades beneficiadas (Bueno-López et al. 2019).

Una forma eficiente de llevar el servicio de electricidad a comunidades alejadas del sistema eléctrico interconectado es a través del concepto de redes aisladas bajo el paradigma de microrredes (Montoya et al. 2019; Talbi et al. 2020). En este escenario, es posible atender a un conjunto de usuarios del servicio de electricidad mediante la interacción de recursos energéticos distribuidos cercanos al punto de conexión de la carga (Martin Serra et al. 2020). Estos recursos energéticos distribuidos en esencia involucran: i) almacenamiento de energía con baterías, ii) sistemas de generación renovable, iii) convertidores electrónicos de potencia, y iv cargas lineales, entre otros.

En la Fig. 1 se presenta una microrred aislada compuesta por un banco de baterías y un sistema de generación fotovoltaica en el lado de corriente continua (CC), la cual se conecta a través de un convertidor fuente de tensión con filtro RLC a un sistema de corriente alterna (CA) para alimentar una carga no lineal (CPL, por sus siglas en inglés). Es importante resaltar que este es el esquema típico para generación de tensión en redes aisladas con alta penetración de recursos energéticos distribuidos (Montoya et al. 2019a; Talbi et al. 2020).

El reto en el sistema que se presenta en la Fig. 1 corresponde al desarrollo de metodologías eficientes de control para mantener la tensión en la carga de forma sinusoidal con magnitud y frecuencia constantes, cumpliendo con los niveles de distorsión armónica permitidos por los estándares internacionales (IEC 2004). Por lo anterior, en este trabajo se propende por revisar y comparar diversos métodos de control aplicables a la regulación de tensión en los terminales de la CPL de la Fig. 1.

Remark 1. Las cargas no lineales que se pueden presentar en una red aislada pueden pueden ser consumos en pequeños motores de inducción, sistemas de iluminación empleando circuitos rectificadores y electrodomésticos, entre otros. 


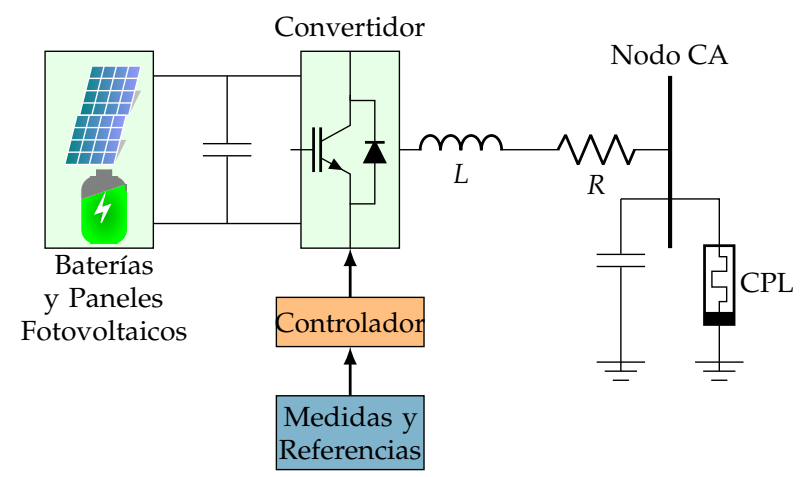

Figure 1. Conexión de una carga no lineal monofásica a un sistema aislado con generación renovable y almacenamiento

\subsection{Motivación}

La necesidad de controlar tensión y frecuencia constante en cargas no lineales con baja distorsión armónica e independiente de las variaciones intrínsecas de la demanda es un reto importante en el área de control de convertidores de potencia (Serra et al. 2017). Un hecho importante en el desarrollo de controladores corresponde a la estabilidad en lazo cerrado, ya que está característica es la garantía esencial de una operación confiable y segura ante diferentes escenarios de demanda (Avila-Becerril and Espinosa-Pérez 2020). En este sentido, esta investigación se motiva en la posibilidad de desarrollar controladores con métodos clásicos y modernos para garantizar estabilidad en lazo cerrado para el sistema dinámico de la Fig. 1. Es de resaltar que la principal complicación al regular tensión y frecuencia en cargas monofásica corresponde al seguimiento de trayectorias variantes en el tiempo (Montoya et al. 2019). Para resolver esta complicación, en este trabajo se propone una representación del sistema en modelo incremental con el que se transforma el problema de seguimiento de trayectorias en un problema de regulación equivalente, tomando como punto de partida la definición de la dinámica admisible (Cisneros et al. 2015).

\subsection{Breve revisión del estado del arte}

En la literatura científica se han propuesto diferentes alternativas para la generación de tensión en redes aisladas monofásicas considerando fuentes de generación de energía renovable y sistemas de almacenamiento de energía mediante convertidores fuente de tensión y filtros LC (Talbi et al. 2020). Algunos de estos trabajos se revisan a continuación: En (Montoya et al. 2019b) y (Montoya et al. 2019a) se presentó el diseño de controladores basados pasividad mediante PI-PBC e IDA-PBC para redes monofásicas aisladas con presencia de sistemas de almacenamiento de energía con supercapacitores, superconductores y baterías, además de fuentes renovables fotovoltaicas y eólicas. La ventaja de estos diseños es la garantía de estabilidad en lazo cerrado mediante la formulación de funciones Hamiltonianias de energía y el teorema de estabilidad de Lyapunov. Este análisis fue extendido en (Montoya et al. 2019) a redes monofásicas con convertidores de tensión y corriente, y cargas de potencia constante garantizando estabilidad en lazo cerrado. Estas estrategias basadas en energía, también se han utilizado en el caso de VSC trifásicos que alimentan cargas aisladas (Serra et al. 2020b), tal como se presenta en (Serra et al. 2016) donde se desarrolla un control IDA-PBC para seguimiento de trayectoria que permite generar tensión con baja distorsión armónica en el caso de alimentar cargas no lineales. Además se muestra una comparación con estrategias IDA-PBC clásicas y controladores PI (Gil-González et al. 2020; Serra et al. 2020c).

Además de las estrategias de control basadas en pasividad antes mencionadas, una gran variedad de controladores han sido diseñados para convertidores de potencia en la aplicación tratada en este trabajo. 
Los autores de (Talbi et al. 2020) presentaron el diseño de un controlador mediante una estrategia de control predictivo considerando cargas lineales y no lineales con validación experimental obteniendo distorsiones armónicas en la tensión de salida menores al 3 \%. En (Nazib et al. 2018) se propone el diseño de un controlador PI mejorado para rechazar perturbaciones para aplicaciones en fuentes de tensión ininterrumpida; sin embargo, la desventaja de esta estrategia de control es que requiere del uso de observadores de fase lo que aumenta el orden del sistema dinámico resultante. Los autores en (Park et al. 2001) presentan un diseño de control mediante regulación de fase en los dispositivos de conmutación forzada. Los resultados experimentales mostraron desempeño adecuado con un índice de distorsión armónica menor al $0.65 \%$ en la tensión de salida. En (Hosseini et al. 2016), se presentó un diseño de control mediante el método directo de Lyapunov para regular la tensión en cargas lineales garantizando estabilidad en lazo cerrado; sin embargo, la desventaja de esta estrategia de diseño es que los autores no presentaron ningún análisis acerca de cargas no lineales.

Con la breve revisión del estado del arte antes mencionada, este artículo se motiva a revisar los principales métodos de control empleados en aplicaciones de redes aisladas desde un punto de vista comparativo mediante la presentación detallada de cada uno de los diseños de control. Esto se hace con el fin de presentar los cálculos necesarios para la obtención de la señal de control. Estas contribuciones se especifican en la siguiente sección.

\subsection{Contribución}

El desarrollo de controladores eficientes para convertidores electrónicos de potencia es de gran interés en la literatura científica, ya que estos corresponden al elemento esencial que realiza la interfaz entre los recursos energéticos distribuidos, los usuarios del servicio de electricidad y las redes de transmisión y/o distribución de energía (Avila-Becerril and Espinosa-Pérez 2020). En el caso de las redes eléctricas destinadas al suministro de energía en redes rurales, este dispositivo (el convertidor) se convierte en el elemento que debe garantizar las tareas de control primario, secundario y terciario en relación con la regulación de tensión y frecuencia independientemente de las variaciones de la carga (Martin Serra et al. 2020). Por lo anterior, en este trabajo de investigación se realiza el diseño de controladores para regular amplitud y frecuencia de la tensión en cargas aisladas lineales y no lineales, con las siguientes contribuciones:

$\checkmark \quad$ Aplicación de técnicas clásicas de control (i.e., control PI, realimentación de estados) y técnicas modernas (i.e., control basado en pasividad) para regular tensión en cargas no lineales con distorsión armónica dentro del rango permitido por las normativas internacionales en relación con la tensión suministrada a los terminales de la carga.

$\checkmark \quad$ Diseño de control garantizando propiedades de estabilidad asintótica considerando como criterio de diseño el lugar geométrico de las raíces.

$\checkmark \quad$ Presentación del diseño de los controladores propuestos de manera didáctica, lo cual es de interés para estudiantes de ingeniería que se están introduciendo en el análisis dinámico de convertidores electrónicos de potencia.

Es importante mencionar que en este trabajo propende por la validación (simulación en software especializado) de técnicas clásicas de control mediante representación en variables de estado para regular tensión en cargas lineales y no lineales; por lo cual se discutirán métodos como control PI, realimentación de estados, control por asignación de interconexión y amortiguamiento basado en pasividad y control PI pasivo; resaltando sus características matemáticas y comparando su desempeño dinámico en términos de la distorsión armónica total. 


\subsection{Organización del documento}

El resto de este documento está organizado de la siguiente forma: en la Sección 2 se presenta la formulación matemática del problema de regulación de tensión en redes monofásicas aisladas mediante una representación promediada del convertidor por fuente de tensión. En la Sección 3 se presentan las derivaciones de cada uno de los controladores estudiados en este trabajo. La Sección 4 presenta las características del sistema de prueba y los diagramas de control implementados. En la Sección 5 se discuten los resultados de simulación y las principales características de implementación. Finalmente, las principales conclusiones derivadas de este trabajo se presentan en la Sección 6.

\section{Formulación del problema de control}

El problema de regulación de tensión en redes eléctricas aisladas monofásicas considerando fuentes renovables y bancos de baterías para atender cargas no lineales con el esquema de conexión de la Fig. 2, considera las siguientes suposiciones:

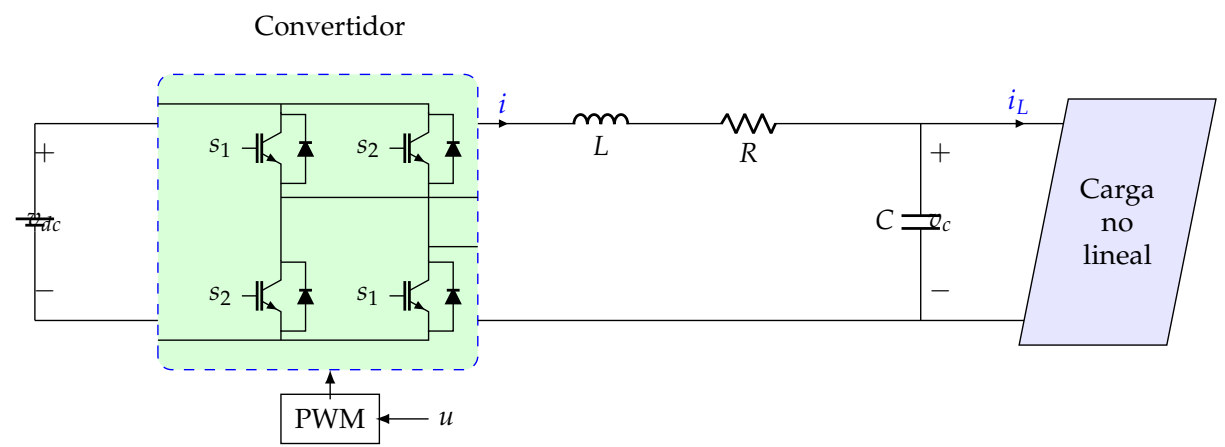

Figure 2. Conexión del convertidor a una carga no lineal

Assumption 1. La corriente de la carga es completamente conocida, i.e., $i_{L}$ es medible y no existe valor de referencia para esta corriente, por lo cual, $i_{L}=i_{L}^{\star}$.

Assumption 2. El lado de CC del convertidor se asume con valor de tensión constante, debido a que existe un banco de baterías en conjunto con una fuente renovable (i.e., generación solar o eólica), de tal forma que su dinámica se puede considerar lenta en relación con el lado de CA.

Assumption 3. Todas las variables de estado son medibles, i.e., corriente en el inductor i y la tensión en la carga $v_{\mathcal{c}}$. Además, los parámetros del filtro RLC son perfectamente conocidos.

Una vez definidas las consideraciones anteriores, el modelo dinámico del sistema presentado en la Fig. 2 se obtiene mediante la aplicación de la primera ley de Kirchhoff donde se conecta el capacitor y la segunda ley de Kirchhoff a la trayectoria cerrada que contiene al inductor. El modelo resultante está dado por,

$$
\begin{aligned}
L \frac{d}{d t} i & =-R i-v_{c}+v_{d c} u, \\
C \frac{d}{d t} v_{c} & =i-i_{L},
\end{aligned}
$$

donde $L, R$ y $C$ son los parámetros de inductancia, resistencia y capacitancia del filtro, respectivamente. Además, $i$ y $v_{c}$ es la corriente a través del inductor y la tensión en el capacitor de salida, respectivamente. 
$v_{d c}$ corresponde a la fuente de tensión del lado de CC. Observe que $u$ representa la señal de control que entra al modulador por ancho de pulso para gobernar el movimiento de apertura y cierre del dispositivos de conmutación forzada dentro del convertidor.

El sistema dinámico (1) y (2) se puede representar de manera compacta mediante un equivalente Hamiltoniano en función de las variables de estado $x_{1}=i$ y $x_{2}=v_{c}$, de la siguiente forma.

$$
\mathcal{D} \dot{x}=[\mathcal{J}-\mathcal{R}] x+g u+\zeta,
$$

donde $\mathcal{D}$ se denomina matriz de inercias del sistema en analogía con los sistemas mecánicos, $\mathcal{J}$ representa la matriz de interconexión la cual es antisimétrica, i.e., $\mathcal{J}=-\mathcal{J}^{T}$ y $\mathcal{R}$ es la matriz conocida como disipación (amortiguamiento natural) del sistema la cual es semidefinida positiva. Además, $g$ es el vector de entrada y $\zeta$ el de perturbaciones externas.

Cada uno de estos términos se calcula como sigue,

$$
\mathcal{J}-\mathcal{R}=\left[\begin{array}{cc}
-R & -1 \\
1 & 0
\end{array}\right], g=\left[\begin{array}{c}
v_{d c} \\
0
\end{array}\right], \mathcal{D}=\left[\begin{array}{ll}
L & 0 \\
0 & C
\end{array}\right], \zeta=\left[\begin{array}{c}
0 \\
i_{L}
\end{array}\right]
$$

Para el desarrollo del control sobre el sistema dinámico (3) es necesario definir la existencia de una trayectoria admisible como sigue:

Definition 1 (Trayectoria admisible). El comportamiento dinámico $x^{\star}$ corresponde a una trayectoria admisible de (3), si este es limitado (acotado), y diferenciable respecto del tiempo, i.e.,

$$
\mathcal{D} \dot{x}^{\star}=[\mathcal{J}-\mathcal{R}] x^{\star}+g u^{\star}+\zeta,
$$

para una señal de control bien definida (acotada) $u^{\star}$.

Ahora, si definimos las variables del modelo incremental $\tilde{x}=x-x^{\star}$ y $\tilde{u}=u-u^{\star}$, y restamos la trayectoria admisible (4) del modelo dinámico (3), se obtiene el modelo incremental siguiente,

$$
\mathcal{D} \dot{\tilde{x}}=[\mathcal{J}-\mathcal{R}] \tilde{x}+g \tilde{u} .
$$

Debido a que algunos de los desarrollos de control analizados en este trabajo, emplean la forma estándar de representación de variables de estado para un sistema lineal, reescribe el modelo dinámico incremental de la forma,

$$
\dot{\tilde{x}}=\mathcal{A} \tilde{x}+\mathcal{B} \tilde{u},
$$

siendo $\mathcal{A}=\mathcal{D}^{-1}[\mathcal{J}-\mathcal{R}]$ y $\mathcal{B}=\mathcal{D}^{-1} g$, respectivamente

Remark 2. Note que el sistema dinámico incremental (6) en la forma estándar es un sistema dinámico lineal de segundo orden, por lo cual se puede regular su salida mediante controladores clásicos como realimentación de estados o control proporcional integral, entre otros. Ya que si el modelo incremental tiende a cero a medida que el tiempo aumenta, entonces las variables de estado $x$ tienden al valor de referencia $x^{\star}$, en otras palabras,

$$
\lim _{t \rightarrow \infty}|\tilde{x}| \rightarrow 0
$$


Remark 3. Nótese que el sistema dinámico definido en (1) y (2) es lineal debido a que se asume que la tensión del lado de CC, i.e., $v_{d c}$ es constante, ya que está es proveída por la combinación de baterías y fuentes de generación renovable como se presentó en la Fig. 1.

Es importante mencionar que si un controlador cumple el objetivo de control (7) este se considera con convergencia asintótica (Avila-Becerril and Espinosa-Pérez 2020), y por tanto válido para regular las variables de estado y alcanzar el seguimiento de trayectoria $x^{\star}$ que regula la tensión deseada en el capacitor de salida de la Fig. 2 en amplitud y frecuencia constante (Avila-Becerril and Espinosa-Pérez 2020).

\section{Desarrollo de los controladores}

En esta sección se explora el desarrollo de controladores clásicos y modernos que garantizan convergencia asintótica de las variables de estado en el modelo incremental a cero. Los controladores desarrollados serán: i) control por asignación de interconexión y amortiguamiento (en adelante IDA-PBC por sus siglas en inglés), ii) control clásico por realimentación de estados, iii) control PI clásico y iv) control pasivo con acción integral (en adelante PI-PBC, por sus siglas en inglés).

Remark 4. El diseño de los cuatro controladores mencionados se basará en el criterio del lugar geométrico de las raíces, con lo cual se podrá garantizar un desempeño dinámico similar mediante el cálculo de las ganancias del controlador por la ubicación de los polos en el lazo cerrado.

\subsection{Diseño IDA-PBC}

El diseño por asignación de interconexión y amortiguamiento basado en pasividad es una técnica de control ampliamente conocida en la literatura especializada (Serra et al. 2017), que puede ser aplicada a sistemas dinámicos lineales y no lineales con la capacidad de garantizar estabilidad asintótica en el sentido de Lyapunov (Ramirez et al. 2009). Para esto, el método IDA-PBC trabaja con un sistema deseado en lazo cerrado en el cual se define la dinámica deseada con la siguiente estructura (Ramirez et al. 2009):

$$
\dot{\tilde{x}}=\left[\mathcal{J}_{d}-\mathcal{R}_{d}\right] \tilde{x}=\mathcal{F}_{d} \tilde{x}
$$

donde $\mathcal{J}_{d} \mathrm{y} \mathcal{R}_{d}$ son las matrices de interconexión y amortiguamiento deseadas que guardan las mismas propiedades de $\mathcal{J}$ y $\mathcal{R}$; siendo $\mathcal{F}_{d}=\mathcal{J}_{d}-\mathcal{R}_{d}$, con la siguiente estructura,

$$
\mathcal{F}_{d}=\left[\begin{array}{cc}
f_{1} & f_{2} \\
-f_{2} & f_{3}
\end{array}\right],
$$

donde $f_{1}, f_{2}$ y $f_{3}$ son constantes reales. Además, el polinomio característico de la matriz $\mathcal{F}_{d}$ toma la siguiente forma,

$$
\lambda^{2}-\left(f_{1}+f_{3}\right) \lambda+f_{2}^{2}+f_{1} f_{3}=0
$$

Con el fin de garantizar que los polos de polinomio característico estén ubicados en el semiplano izquierdo del plano complejo, este es comparado con el polinomio deseado en lazo cerrado $\lambda^{2}+$ $\left(p_{1}+p_{3}\right) \lambda+p_{1} p_{2}=0$, siendo $p_{1}$ y $p_{2}$ los polos deseados, tal que real $\left(p_{1}, p_{2}\right)>0$. 
Comparando (10) con el polinomio característico en lazo cerrado, se tiene el siguiente sistema de ecuaciones:

$$
\begin{array}{r}
f_{1}+f_{3}=-p_{1}-p_{2} \\
f_{1} f_{3}+f_{2}^{2}=p_{1} p_{2} .
\end{array}
$$

Remark 5. El sistema de ecuaciones dado en (11) tiene infinitas soluciones, ya que es un sistema algebraico lineal con un grado de libertad adicional (i.e., $f_{2}$ ); por lo cual existen múltiples coeficientes en $\mathcal{F}_{d}$ que cumplen con las condiciones de los polos en lazo cerrado, y por tanto hacen estable el sistema dinámico incremental.

Nótese que una posible solución del sistema (11), puede ser la siguiente:

$$
\begin{aligned}
& f_{1}=\alpha p_{1}, \\
& f_{3}=-(1+\alpha) p_{1}-p_{2} . \\
& f_{2}=\sqrt{(1+\alpha)\left(p_{1} p_{2}+\alpha p_{1}^{2}\right)},
\end{aligned}
$$

siendo $\alpha>0$, con $p_{1} p_{2}+\alpha p_{1}^{2}>0$. Por lo cual, los polos $p_{1}$ y $p_{2}$ se asignan únicamente reales.

Para obtener la ley de control empleando el método IDA-PBC se comparan el sistema dinámico incremental en lazo abierto (6) con el sistema dinámico incremental en lazo cerrado deseado (8), con lo cual se obtiene:

$$
\begin{aligned}
\mathcal{F}_{d} \tilde{x} & =\mathcal{A} \tilde{x}+\mathcal{B} u \\
\mathcal{B}^{T} \mathcal{B} u & =\mathcal{B}^{T}\left[\mathcal{F}_{d}-\mathcal{A}\right] \tilde{x} \\
u & =\left(\mathcal{B}^{T} \mathcal{B}\right)^{-1}\left(\mathcal{B}^{T}\left[\mathcal{F}_{d}-\mathcal{A}\right]\right) \tilde{x} .
\end{aligned}
$$

Nótese que la ley de control (13) es una realimentación de variables de estado de la forma $\tilde{u}=-k_{\text {IDA }}^{T} \tilde{x}$, siendo

$$
k_{\mathrm{IDA}}^{T}=-\left(\mathcal{B}^{T} \mathcal{B}\right)^{-1}\left(\mathcal{B}^{T}\left[\mathcal{F}_{d}-\mathcal{A}\right]\right)
$$

\subsection{Diseño por realimentación de estados}

El control por realimentación de estados, también conocido como asignación de polos, es una de las técnicas de control más empleadas en sistemas lineales (Serra et al. 2020a; ?), ya que permite diseñar una ley de control mediante realimentación negativa de cada uno de los estados de la planta y realizar regulación de estas (Fadali and Visioli 2013). Para el diseño de un controlador mediante este método, se considera la aproximación clásica utilizando la fórmula de Ackermann. Por lo cual se considera una señal de control de la forma $\tilde{u}=k_{\mathrm{R}}^{T} \tilde{x}$, tal que el sistema dinámico incremental (6) en lazo cerrado toma la forma:

$$
\tilde{x}=\left[\mathcal{A}-\mathcal{B} k_{\mathrm{R}}^{T}\right] \tilde{x}
$$

Según la teoría clásica de control, para el sistema de segundo orden estudiado en este trabajo, el cálculo del vector de ganancias de realimentación $k_{\mathrm{R}}^{T}$ según la formulación de Ackermann adquiere la siguiente representación:

$$
k_{\mathrm{R}}^{T}=\left[\begin{array}{ll}
0 & 1
\end{array}\right] \mathcal{C}^{-1} \Delta_{\text {Nuevo }}(\mathcal{A}),
$$


siendo $\mathcal{C}$ la matriz de controlabilidad del sistema, i.e., $\mathcal{C}=[\mathcal{B} \mathcal{B} \mathcal{A}] ;$ y $\Delta_{\text {Nuevo }}(\mathcal{A})$ representa el polinomio característico deseado en la matriz $\mathcal{A}$, es decir,

$$
\Delta_{\text {Nuevo }}(\mathcal{A})=\mathcal{A}^{2}+\left(p_{1}+p_{3}\right) \mathcal{A}+p_{1} p_{2} \mathcal{I}
$$

con $\mathcal{I}$ una matriz identidad de dimensiones apropiadas.

Remark 6. La ley de control por realimentación de estados garantiza estabilidad asintótica del sistema ya que reasigna la parte real de los polos al semiplano complejo izquierdo, lo que implica que pasado el tiempo t, estas dinámicas desaparecerán y sólo quedará la respuesta forzada del sistema, i.e., la respuesta definida por $u^{\star}$.

\subsection{Diseño PID clásico}

El diseño de un control clásico empleando acciones proporcionales e integrales, es quizá el método más empleado en la literatura para sistemas lineales, ya que permite un diseño simple y con capacidad de eliminar el error de estado estacionario, incluso en presencia de incertidumbre paramétrica. Para el diseño clásico de control PI considere la representación en función de transferencia de la variable de interés y la ley de control en 6, como sigue:

$$
\mathcal{H}(s)_{\mathrm{LA}}=\frac{\tilde{x}_{2}(s)}{\tilde{u}(s)}=\frac{\beta_{0}}{s^{2}+\beta_{1} s+\beta_{2}},
$$

$\operatorname{con} \beta_{0}=\frac{v_{d c}}{L C}, \alpha_{1}=\frac{R}{L}$ y $\alpha_{2}=\frac{1}{L C}$, respectivamente.

Si se considera el error de seguimiento como $e=r-\tilde{x}_{2}$, donde $r$ es la referencia deseada, se puede definir un controlador clásico PID de la forma $\tilde{u}=k_{p}^{\mathrm{PID}} e+k_{i}^{\mathrm{PID}} \int e d t+k_{d}^{\mathrm{PID}} \frac{d}{d t} e$. Ahora, si se aplica la transformada de Laplace para obtener la función de transferencia en lado cerrado $\mathcal{H}(s)_{\mathrm{LC}}=\frac{\tilde{x}_{2}(s)}{\tilde{r}(s)}$ como:

$$
\mathcal{H}(s)_{\mathrm{LC}}=\frac{\beta_{0}\left(k_{d}^{\mathrm{PID}} s^{2}+k_{p}^{\mathrm{PID}} s+k_{i}^{\mathrm{PID}}\right)}{s^{3}+\left(\beta_{1}+\beta_{0} k_{d}^{\mathrm{PID}}\right) s^{2}+\left(\beta_{2}+\beta_{0} k_{p}^{\mathrm{PID}}\right) s+\beta_{0} k_{i}^{\mathrm{PID}}}
$$

Remark 7. Para ajustar las ganancias del controlador (i.e., $k_{p}^{P I D}, k_{i}^{P I D}$ y $k_{d}^{P I D}$ ), se realiza la comparación del polinomio característico en lazo cerrado de la función de transferencia (18) con el polinomio característico deseado:

$$
p_{d}(s)=s^{3}-\left(p_{1}+p_{2}+p_{3}\right) s^{2}
$$

$$
+\left(p_{1} p_{2}+p_{1} p_{3}+p_{2} p_{3}\right) s-p_{1} p_{2} p_{3}
$$

donde $p_{1}, p_{2}$ y $p_{3}$ son los polos deseados del sistema en lazo cerrado, siendo para el caso de esta investigación números reales negativos.

Al comparar el polinomio deseado $p_{d}(s)$ con el denominador de la función de transferencia en lazo cerrado, se obtienen las siguientes ganancias para el controlador PID

$$
\begin{aligned}
k_{d}^{\mathrm{PID}} & =-\frac{p_{1}+p_{2}+p_{3}+\beta_{1}}{\beta_{0}}, \\
k_{i}^{\mathrm{PID}} & =-\frac{p_{1} p_{2} p_{3}}{\beta_{0}}, \\
k_{p}^{\mathrm{PID}} & =\frac{p_{1} p_{2}+p_{1} p_{3}+p_{2} p_{3}-\beta_{2}}{\beta_{0}} .
\end{aligned}
$$


Remark 8. Es de destacar que en la práctica, la implementación de controladores derivativos para controlar convertidores de potencia debe ser considerado con suma cautela, debido a que ello requiere el procesamiento de señales conmutadas de alta frecuencia cuya derivada puede producir señales altamente ruidosas que vayan en detrimento del objetivo de control deseado (Yu 2018).

\subsection{Diseño PI-PBC}

El diseño de controladores basados en pasividad con acción proporcional integral corresponden a una alternativa eficiente para sistemas dinámicos lineales o sistemas dinámicos bilineales (Cisneros et al. 2015). Este método se basa en la búsqueda de la salida pasiva natural del sistema, i.e., ỹ. Esta salida tiene la forma $\tilde{y}=g^{T} \tilde{x}$. Si se considera la salida pasiva, se tiene que la ley de control que estabiliza el sistema en lazo cerrado es,

$$
\begin{aligned}
& \tilde{u}=-k_{p} \tilde{y}+k_{i} z, \\
& z=-\tilde{y},
\end{aligned}
$$

Para demostrar que la ley de control (20) hace al sistema dinámico incremental (5) estable en el sentido de Lyapunov, considérese la función candidata de Lyapunov,

$$
\mathcal{V}(\tilde{x})=\frac{1}{2} \tilde{x}^{T} \mathcal{D} \tilde{x}+\frac{1}{2} z^{T} k_{i} z
$$

donde $\mathcal{V}(\tilde{x})$ es una función definida positiva tal que $\mathcal{V}(0)=0$, y $\mathcal{V}(\tilde{x})>0, \quad \forall \tilde{x} \neq 0$, con lo cual se cumplen las primeras dos condiciones del teorema de estabilidad de Lyapunov (Perko 2013).

Ahora, si se toma la derivada temporal de (21), se obtiene lo siguiente,

$$
\dot{\mathcal{V}}(\tilde{x})=\frac{1}{2} \tilde{x}^{T} \mathcal{D} \dot{\tilde{x}}+\frac{1}{2} z^{T} k_{i} \dot{z}
$$

luego, si se sustituye (5) en (22), y recordando que $\mathcal{J}$ es una matriz antisimétrica, se llega al siguiente resultado,

$$
\dot{\mathcal{V}}(\tilde{x})=-\tilde{x}^{T} \mathcal{R} \tilde{x}+\tilde{x}^{T} g\left[-k_{p} \tilde{y}+k_{i} z\right]-z^{T} k_{i} \tilde{y} .
$$

Considerando que $\tilde{y}^{T}=\tilde{x}^{T} g$, y que $k_{i}$ es un parámetro escalar, entonces (23) puede ser simplificado como,

$$
\dot{\mathcal{V}}(\tilde{x})=-\tilde{x}^{T} \mathcal{R} \tilde{x}+\tilde{y}^{T} k_{p} \tilde{y}<0
$$

Remark 9. La expresión (24) es definida negativa, ya que $\mathcal{R}$ es definida positiva, con lo cual el sistema dinámico incremental (5) es asintóticamente estable en el sentido de Lyapunov. Esto implica que el objetivo de control definido en (7) se cumple plenamente.

Es importante mencionar que las ganancias $k_{i}$ y $k_{p}$ son constantes positivas y se diseñan tal que el desempeño dinámico en lazo cerrado sea equivalente al modelo PID clásico. Además, en este caso, la salida pasiva $\tilde{y}$ toma la forma $v_{d c} \tilde{x}_{1}$, por lo cual no depende de la medida directa de la tensión en el punto de conexión de la carga, siendo esta una diferencia crucial con respecto a los enfoques de IDA-PBC y de realimentación de estado. 


\section{Sistema de prueba e implementación}

Para la validación numérica del sistema de prueba se consideran los parámetros reportados en la Tabla 1. Además, en la Fig. 3 se presenta la conexión eléctrica de la carga no lineal.

Table 1. Parámetros del sistema de conversión empleados en las simulaciones

\begin{tabular}{cccccc}
\hline Parámetro & Valor & Unidad & Parámetro & Valor & Unidad \\
\hline$v_{d c}$ & 380 & $\mathrm{~V}$ & $L_{1}$ & 1 & $\mathrm{mH}$ \\
$L$ & 1 & $\mathrm{mH}$ & $R_{1}$ & 10 & $\Omega$ \\
$R$ & 100 & $\mathrm{~m} \Omega$ & $C_{1}$ & 550 & $\mu \mathrm{F}$ \\
$C$ & 150 & $\mu \mathrm{F}$ & $R_{2}$ & 50 & $\Omega$ \\
\hline
\end{tabular}

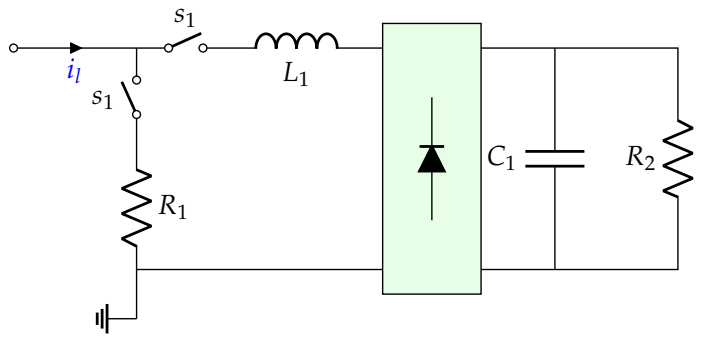

Figure 3. Conexión de la carga al convertidor

La lógica de operación de la carga se presenta a continuación, considerando que el tiempo de simulación es $t_{\text {sim }}=400 \mathrm{~ms}$ :

$\checkmark \quad$ Para la carga no lineal se considera que en el tiempo $t=0 \mathrm{~s}$ el rectificador está conectado, luego en el tiempo $t=0.25 t_{\text {sim }}$ este se desconecta, y finalmente es reconectado en el tiempo $t=0.625 t_{\text {sim }}$.

$\checkmark \quad$ La carga resistiva se inicia desconectada y en el tiempo $t=0.25 t_{\text {sim }}$ esta es conectada a la red.

La idea general con este comportamiento propuesto de la carga es analizar durante 20 ciclos de la señal de salida $(f=50 \mathrm{~Hz})$, el efecto de tener cargas no lineales, cargas puramente resistivas y la combinación de ambas.

\subsection{Implementación de la planta y señales de referencia}

La implementación de la planta es realizada en el software MATLAB en el entorno de Simulink considerando la estructura presentada en la Fig. 1 y la carga no lineal del Fig. 3.

En relación con el objetivo de control, debido a que la aplicación proyectada es de abastecimiento de energía en redes rurales, se selecciona una tensión de distribución típico de baja tensión como sigue:

$$
x_{2}^{\star}(t)=120 \sqrt{2} \sin (100 \pi t) .
$$

Un hecho importante en los desarrollos de control propuesto es que estos han sido aplicados para hacer que el sistema dinámico incremental tienda a cero en el menor tiempo posible a través de la señal de control $\tilde{u}$; sin embargo, la señal de control $u$ aplicable a la planta es la suma de $\tilde{u}+u^{\star}$, por lo cual es necesario determinar el valor de $u^{\star}$. Esta señal de control puede ser obtenida a partir de las trayectorias admisibles definidas en (4). Al resolver este sistema de ecuaciones se obtiene lo siguiente:

$$
u^{\star}=\frac{1}{v_{d c}}\left(L \frac{x_{1}^{\star}}{d t}+R x_{1}^{\star}+x_{2}^{\star}\right),
$$




$$
x_{1}^{\star}=C \frac{d}{d t} x_{2}^{\star}+i_{L}
$$

\subsection{Implementación de los controladores}

Para la implementación de cada uno de los controladores es importante mencionar que: $i$ ) los diseños IDA-PBC y realimentación de estados requiere de las medidas de tensión $\left(x_{2}\right)$ y corriente $x_{1}$ para el cálculo de la señal de control; ii) la estrategia PID requiere solo de la medición de la tensión de salida, i.e., $x_{2} ; \mathrm{y}$ iii) el diseño PI-PBC solo requiere de la medición de la corriente, i.e., $x_{1}$ para lograr el objetivo de control. Lo anterior es principalmente importante en aplicaciones reales en redes aisladas ya que permitirá la reducción de costos de diseño debido a la reducción en el número de sensores requeridos.

Por otro lado, las ganancias de control empleadas en estas implementaciones se presentan la Tabla 2 y han sido calculadas considerando la ubicación de los polos deseados reales $p_{1}=-4000, p_{2}=-5000 \mathrm{y}$ $p_{3}=-9000$, respectivamente.

Table 2. Ganancias para cada uno de los controladores

\begin{tabular}{lll}
\hline Método & & \multicolumn{1}{c}{ Ganancias } \\
\hline IDA-PBC & $k_{\mathrm{IDA}_{1}}=0.131316$ & $k_{\mathrm{IDA}_{2}}=0.117098$ \\
Realmentación & $k_{\mathrm{R}_{1}}=0.023421$ & $k_{\mathrm{R}_{2}}=0.005263$ \\
PI-PBC & $k_{p}=0.01000$ & $k_{p}=0.001000$ \\
PID & $k_{p}^{\mathrm{PID}}=0.0372368$ & $k_{i}^{\mathrm{PID}}=71.052631 \quad k_{d}^{\mathrm{PID}}=7.065789 \times 10^{-6}$ \\
\hline
\end{tabular}

\section{Resultados de simulación}

En esta sección se presentan los resultados de simulación correspondientes a cada uno de los controladores, considerando un tiempo de muestreo de $1 \mu \mathrm{s} \mathrm{y} \mathrm{una} \mathrm{frecuencia} \mathrm{de} \mathrm{conmutación} \mathrm{de} 10 \mathrm{kHz}$.

A manera de ilustración se selecciona el diseño de control IDA-PBC ${ }^{1}$ para mostrar el comportamiento de las variables de interés, i.e., tensión de salida $x_{2}$, corriente del filtro $x_{1}$ y corriente de carga. En la Fig. 4 se presentan los resultados del diseño del control IDA-PBC y el error de seguimiento de los diferentes controladores.

De la Fig. 4 se puede observar que:

$\checkmark \quad$ La tensión en los terminales de la carga tiene forma sinusoidal y sigue de manera adecuada la referencia en términos de la magnitud y frecuencia deseadas como se definió en la Ecuación (25). Nótese que las variaciones promedio en frecuencia son menores a $0.02 \mathrm{~Hz}$ para todos los controladores, siendo el PID el que presenta extremos de hasta $0.06 \mathrm{~Hz}$. Sin embargo, este comportamiento en la salida de frecuencia se debe en parte a la dinámica interna del observador de fase empleado para su medición, el cual puede introducir errores de estimación que resultan en oscilaciones inexistentes en la señal de salida (Martin Serra et al. 2020).

$\checkmark \quad$ En relación con el error de seguimiento, este se ha calculado como $E_{r}=\frac{x_{2}-x_{2}^{\star}}{120 \sqrt{2}}$ y refleja que: el controlador PID tiene oscilaciones en la estimación con promedio del $5 \%$; el controlador por realimentación de estados presenta menor error de estimación, con variaciones promedio de $1 \%$; mientras que los controladores basados en pasividad, i.e., IDA- y PI-PBC, presentan el mismo desempeño dinámico en términos de seguimiento de referencia con oscilaciones en promedio de hasta $2 \%$ al inicio de la simulación y que se reducen hasta $1 \%$ al final de la misma.

1 Nótese que esta selección es arbitraria y se escoge meramente con fines ilustrativos 
$\checkmark \quad$ El comportamiento de la corriente sobre el filtro de salida y sobre la carga evidencian un comportamiento de acuerdo al tipo de carga que está siendo alimentada por el sistema y se puede observar que: $i$ ) durante el primer periodo $0 \mathrm{~ms} \leq t \leq 100 \mathrm{~ms}$ la carga es completamente no lineal ya que sólo está conectado el rectificador a la salida de la planta, por ende la corriente se comporta de forma no lineal; ii) durante el período de tiempo comprendido entre $100 \mathrm{~ms}$ y $250 \mathrm{~ms}$ la carga es lineal y por lo tanto la corriente es sinusoidal pura debido a la presencia de una única carga resistiva; y iii) para el período de tiempo mayor a $250 \mathrm{~ms}$ la carga se compone de una carga resistiva y una carga no lineal, y por lo tanto el comportamiento de la corriente es acorde a este tipo de carga. Este escenario de simulación permite concluir que todos los diseños de control tienen la capacidad de regular la tensión de salida en la carga independiente de las variaciones de esta, lo cual es ideal para aplicaciones en redes aisladas en las que no se conoce la composición real de la carga y por tanto se requiere de estrategias de control robustas.

Con el fin de comparar de mejor manera los controladores propuestos para cada uno de los casos considerados se obtiene el índice de distorsión armónica total (THD, por sus siglas en inglés) para la tensión de salida. Estos valores se reportan en la Tabla 3.

Table 3. Distorsión armónica de la señal de tensión proveída a la carga

\begin{tabular}{lcccc}
\hline Método & IDA-PBC & Realmentación & PI-PBC & PID \\
\hline THD [\%] & 0.40 & 0.56 & 0.13 & 0.24 \\
\hline
\end{tabular}

Al observar los índices de distorsión armónica de la Tabla 3 es posible concluir que el diseño de PI-PBC es el que tiene mejor comportamiento dinámico en la tensión de salida dado que este es el menor de los cuatro métodos comparativos. Sin embargo, es importante resaltar que el método de realimentación de estados, aunque presenta un menor error de seguimiento y un comportamiento en frecuencia con menor oscilación (ver Fig. 4), presenta la mayor distorsión armónica debido al ruido de alta frecuencia presente en el seguimiento de la señal de referencia.

\section{Conclusiones y trabajos futuros}

En este artículo se presentó el desarrollo de cuatro controladores basados en el modelo para el problema de regulación de amplitud y frecuencia de la tensión de salida en redes eléctricas aisladas con carga no lineal empleando un convertidor por fuente de tensión alimentado por un conjunto de generación renovable y baterías que garantizan tensión constante en el lado de CC del convertidor. En este desarrollo se comparan métodos basados en pasividad como PI-PBC e IDA-PBC con métodos clásicos como realimentación estados y PID. Para realizar análisis comparativo se ajustaron las ganancias de control con el fin de obtener la misma reubicación de polos en lazo cerrado.

Los resultados de simulación muestran que cada uno de los controladores propuestos tienen la capacidad de regular amplitud y frecuencia de la tensión de salida de manera correcta garantizando baja distorsión armónica (THD $\leq 0.60 \%$ ) independientemente de las variaciones de la carga, siendo el control PI-PBC el de mejor desempeño con $0.13 \%$ y la realimentación estados clásica el de peor desempeño con $0.56 \%$.

En el desarrollo de los controladores se evidenció que los métodos IDA-PBC y realimentación estados requieren de la medida de las dos variables de estado, i.e., $x_{1}=i$ y $x_{2}=v_{\mathcal{C}}$, para ejecutar la tarea de control; mientras los métodos PI-PBC y PID requieren sólo de un estado, i.e., $x_{1}$ para el PI-PBC y $x_{2}$ para el PID. Lo cual los hace más atractivos desde el punto de vista de implementación en relación con la reducción en los costos de diseño del control. Sin embargo, como se mencionó en parágrafos anteriores, la implementación práctica de un control PID en sistemas conmutados puede incurrir en malfuncionamiento 

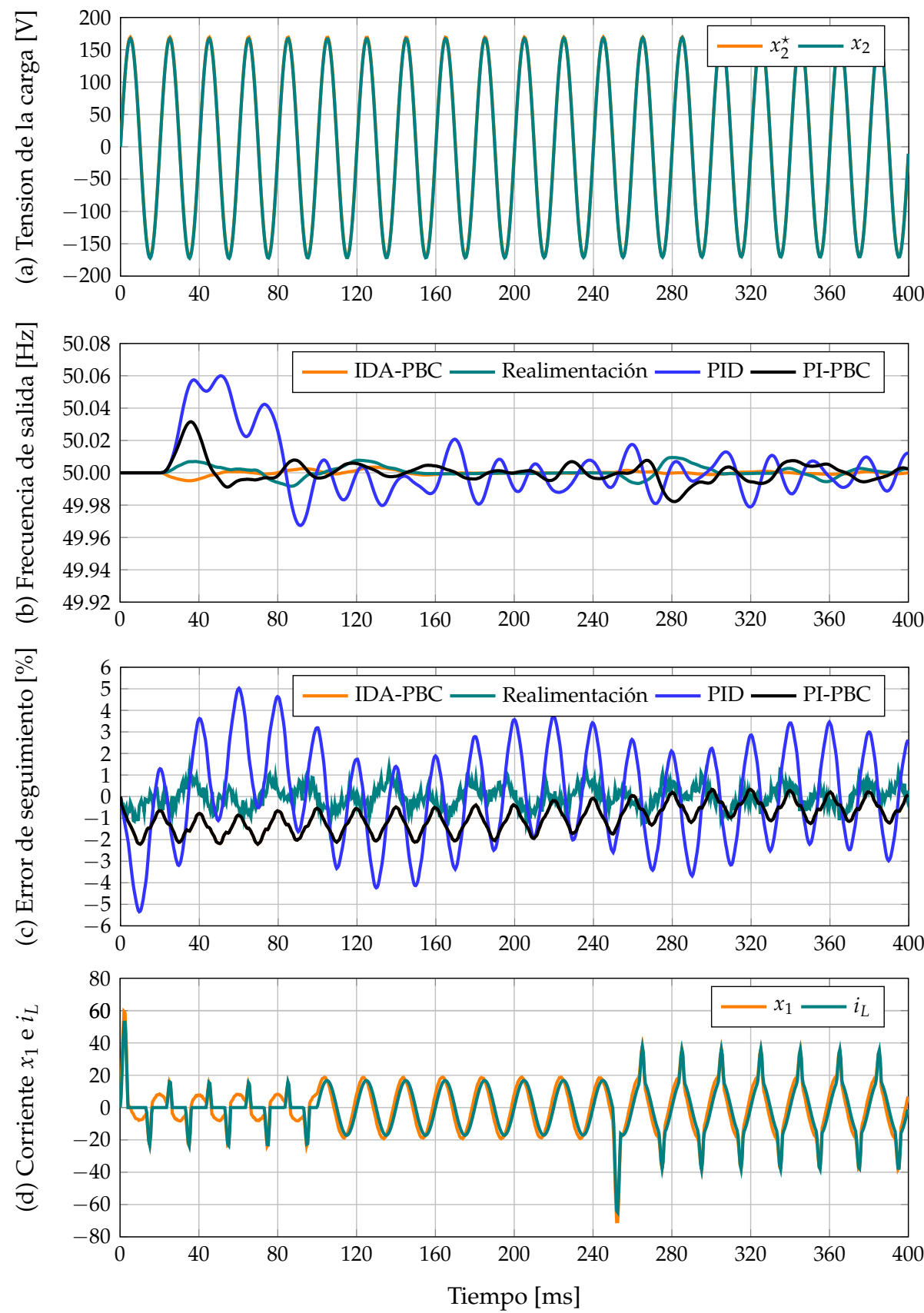

Figure 4. Comportamiento del controlador IDA-PBC y comparación de desempeño con los demás controladores: (a) tensión de alimentación de la carga, (b) frecuencia de la tensión de salida, (c) error porcentual de seguimiento de tensión, y (d) corriente de salida del filtro y entregada a la carga

del control debido a la amplificación de ruido en alta frecuencia producido por la parte derivativa del controlador.

Como trabajos futuros se proponen los siguientes: $i$ ) la comparación de métodos de control como lógica difusa, redes neuronales y planos deslizantes para la regulación de tensión y frecuencia para cargas no lineales en zonas aisladas; ii) el desarrollo de estimadores de estado para las variables del sistema 
con el fin de reducir costos de implementación; y iii) la extensión de la red aislada múltiples nodos interconectados con redes de distribución local y presencia de diferentes fuentes de almacenamiento de energía.

Author Contributions: Conceptualización, W.G.-G.; Metodología, W.G.-G. y S.Y.B; Investigación, W.G.-G. y S.Y.B; Escritura, revisión y Edición, W.G.-G., S.Y.B., F.M., M.B.-L., y G.M.

\section{References}

Abu-Rub, H., Malinowski, M., and Al-Haddad, K., editors (2014). Power Electronics for Renewable Energy Systems, Transportation and Industrial Applications. John Wiley \& Sons, Ltd. doi:10.1002/9781118755525.

Avila-Becerril, S. and Espinosa-Pérez, G. (2020). Control of islanded microgrids considering power converter dynamics. International Journal of Control, (just-accepted):1-19.

Bueno-Lopez, M. and Garzon Lemos, S. (2017). Electrification in non-interconnected areas: Towards a new vision of rurality in colombia. IEEE Technology and Society Magazine, 36(4):73-79.

Bueno-López, M., Rodríguez-Sánchez, P., and Molinas, M. (2019). Sustainable model for rural electrification projects in non-interconnected areas in colombia. In 2019 IEEE Global Humanitarian Technology Conference (GHTC), pages 1-6. Cisneros, R., Pirro, M., Bergna, G., Ortega, R., Ippoliti, G., and Molinas, M. (2015). Global tracking passivity-based PI control of bilinear systems: Application to the interleaved boost and modular multilevel converters. Control Engineering Practice, 43:109-119.

Ellabban, O., Abu-Rub, H., and Blaabjerg, F. (2014). Renewable energy resources: Current status, future prospects and their enabling technology. Renewable Sustainable Energy Rev., 39:748-764. doi:10.1016/j.rser.2014.07.113.

Fadali, M. S. and Visioli, A. (2013). Digital control engineering: analysis and design. Academic Press.

Gil-González, W., Martin Serra, F., Montoya, O. D., Ramírez, C. A., and Orozco-Henao, C. (2020). Direct power compensation in ac distribution networks with sces systems via pi-pbc approach. Symmetry, 12(4):666.

Hosseini, S. K., Mehrasa, M., Taheri, S., Rezanejad, M., Pouresmaeil, E., and Catalao, J. P. S. (2016). A control technique for operation of single-phase converters in stand-alone operating mode. In 2016 IEEE Electrical Power and Energy Conference (EPEC). IEEE. doi:10.1109/epec.2016.7771739.

IEC, I. (2004). 62040-3: Uninterruptible power systems (ups)-part 3: Method of specifying the performance and test requirements. Switzerland: IEC.

Martin Serra, F., Fernández, L., Montoya, O. D., Gil-González, W., and Hernández, J. (2020). Nonlinear Voltage Control for Three-Phase DC-AC Converters in Hybrid Systems: An Application of the PI-PBC Method. Electronics, 9:847.

Montoya, O. D., Garces, A., Avila-Becerril, S., Espinosa-Pérez, G., and Serra, F. M. (2019). Stability Analysis of Single-Phase Low-Voltage AC Microgrids With Constant Power Terminals. IEEE Trans. Circuits Syst. II, 66(7):1212-1216.

Montoya, O. D., Gil-González, W., Avila-Becerril, S., Garces, A., and Espinosa-Pérez, G. (2019a). Integración de REDs en Redes AC: una Familia de Controladores Basados en Pasividad. Revista Iberoamericana de Automática e Informática industrial, 16(2):212. doi:10.4995/riai.2018.10666.

Montoya, O. D., Gil-González, W., and Garces, A. (2019b). Distributed energy resources integration in single-phase microgrids: An application of IDA-PBC and PI-PBC approaches. Int. J. Electr. Power Energy Syst., 112:221-231. doi:10.1016/j.ijepes.2019.04.046.

Nazib, A. A., Holmes, D. G., and McGrath, B. P. (2018). High Quality Voltage Regulation of Single Phase Autonomous Microgrids Under Nonlinear Load Conditions. In 2018 IEEE Energy Conversion Congress and Exposition (ECCE), pages 5169-5176.

Parhizi, S., Lotfi, H., Khodaei, A., and Bahramirad, S. (2015). State of the Art in Research on Microgrids: A Review. IEEE Access, 3:890-925.

Park, H.-W., Park, S.-J., Park, J.-G., and Kim, C.-U. (2001). A novel high-performance voltage regulator for single-phase AC sources. IEEE Trans. Ind. Electron., 48(3):554-562. doi:10.1109/41.925582.

Perko, L. (2013). Differential equations and dynamical systems, volume 7. Springer Science \& Business Media. 
Ramirez, H., Sbarbaro, D., and Ortega, R. (2009). On the control of non-linear processes: An IDA-PBC approach. Journal of Process Control, 19(3):405-414.

Serra, F., Doria-Cerezo, A., De Angelo, C., Fernández, L. M., and Bodson, M. (2020a). Complex pole placement control for a three-phase voltage source converter. In 2020 IEEE International Conference on Industrial Technology (ICIT), pages 901-906. IEEE.

Serra, F. M., Angelo, C. H. D., and Forchetti, D. G. (2016). IDA-PBC control of a DC-AC converter for sinusoidal three-phase voltage generation. Int. J. Electron., 104(1):93-110. doi:10.1080/00207217.2016.1191087.

Serra, F. M., De Angelo, C. H., and Forchetti, D. G. (2017). IDA-PBC control of a DC-AC converter for sinusoidal three-phase voltage generation. International Journal of Electronics, 104(1):93-110.

Serra, F. M., Doria-Cerezo, A., Angelo, C. H. D., Fernandez, L. L. M., and Bodson, M. (2020b). Complex Pole Placement Control for a Three-Phase Voltage Source Converter. In 2020 IEEE International Conference on Industrial Technology (ICIT). IEEE. doi:10.1109/icit45562.2020.9067255.

Serra, F. M., Fernández, L. L. M., Montoya, O. D., Gil-González, W. J., and Hernández, J. C. (2020c). Nonlinear Voltage Control for Three-Phase DC-AC Converters in Hybrid Systems: An Application of the PI-PBC Method. Electronics, 9(5):847. doi:10.3390/electronics9050847.

Talbi, B., Krim, F., Laib, A., and Sahli, A. (2020). Model predictive voltage control of a single-phase inverter with output LC filter for stand-alone renewable energy systems. Electrical Engineering. doi:10.1007/s00202-020-00936-5. $\mathrm{Yu}, \mathrm{W}$. (2018). PID Control with Neural Compensation. In PID Control with Intelligent Compensation for Exoskeleton Robots, pages 81-107. Elsevier. doi:10.1016/b978-0-12-813380-4.00005-0.

(C) 2020 by the authors. Licensee TESEA, Cartagena, Colombia. This article is an open access article distributed under the terms and conditions of the Creative Commons Attribution (CC BY) license (http://creativecommons.org/licenses/by/4.0/). 